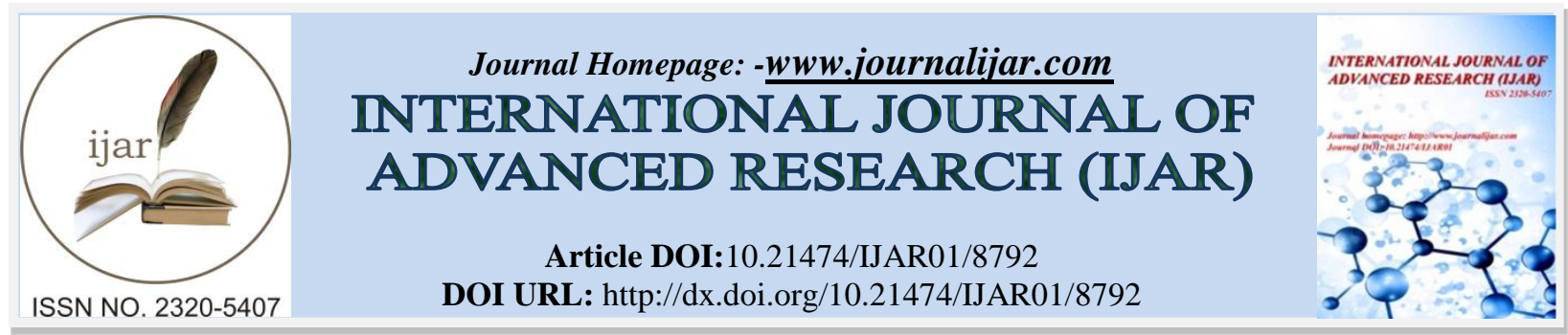

RESEARCH ARTICLE

\title{
SILK FIBROIN IN EFFECTIVE WOUND DRESSING: AN OVERVIEW.
}

\section{Shailendra Singh Shera ${ }^{1}$ and Nilanjan Das ${ }^{2}$.}

1. Department of Biochemical Engineering, Indian Institute of Technology (Banaras Hindu University) Varanasi Campus, Varanasi- 221005, India.

2. Nalanda Foundation, New Delhi-20, India.

\section{Manuscript Info}

Manuscript History

Received: 03 February 2019

Final Accepted: 05 March 2019

Published: April 2019

Key words:-

Wound, dressing, silk fibroin, health care, SDG.

\section{Abstract}

Silk materials have been shown to promote wound healing since the 1990s. Both fibroin and sericin have been found to be an effective substrate for the proliferation of adherent animal cells and can be used as a substitute for collagen. Because of their excellent physical and biological properties, silk fibroin and sericin were widely investigated for their use in biomedical applications including wound healing materials.

Medical wound dressing is a commonly-used treatment for skin defects. Silk fibroin (SF) is a natural protein derived from Bombyx mori cocoons, and shows potential in tissue repair applications due to its excellent biomedical properties. Numerous silk fibroin wound dressings have been developed in the lab, however, lack of large animal studies and clinical trials have hindered their wide use in the clinic.

In recent era, the growing field of tissue engineering has introduced remarkable wound dressings based on natural polymers. The unique properties of SF, such as its biocompatibility, biodegradability, high water and oxygen uptake, low immunogenicity, and robust mechanical properties, make it an exceptional choice for wound healing.

Despite the successful use of SF in wound dressings, it is not yet approved as an artificial skin.

Recently, biomimetic wound dressings were introduced as potential replacements for treating skin injuries. Although there are some clinically available skin replacements, the range of wound types and locations necessitates a broader range of options for the clinic. Natural polymeric-based dressings are of central interest in this area due to their outstanding biocompatibility, biodegradability, low toxicity, and nonallergenic nature. Among them, silk fibroin has exceptional characteristics as a wound dressing. SF-based dressings can also be used as carriers for delivering drugs, growth factors, and bioactive agents to the wound area, while providing appropriate support for complete healing. This dressing is expected to promote good health and wellbeing of masses at an affordable cost. Therefore this is in alignment to UN sustainable development goal (SDG) and can be very effective for smart Kashi initiatives.

Copy Right, IJAR, 2019,. All rights reserved. 


\section{Introduction:-}

Human skin acts as an external defense system shielding our inner structures from external harsh environment as well as from several microorganisms. In human, adult skin is composed of three layers; epidermis or stratum corneum consisting of keratinocytes, dermis (collagen rich connective tissue) and hypodermis or fat tissue rich subcutaneous layer providing thermal isolation and mechanical protection to the body [1-4]. Wound is a type of injury which happens relatively quickly where skin is torn, cut, or punctured (an open wound), or where blunt force trauma causes a contusion (a closed wound). However, in pathology, wound specifically refers to a sharp injury which damages the dermis of the skin. Wounds might be due to physicochemical or thermal damage. Acute wounds need a healing period over 8-12 weeks, (e.g., burns, chemical injuries, cuts) whereas chronic wounds might be a reflection of diseases, such as venous or arterial vascular insufficiency, pressure necrosis, cancer, and diabetes requiring much longer healing time (weeks-months to years) and often persist in an inflamed condition and fail to reach a normal healthy state [5-7]. Therefore, delayed or impaired wound healing poses a serious threat worldwide. Infringing the integral structure of skin causes cutaneous wounds which are prone to infection thus very difficult to heal. Millions of people across the world suffer from contaneous wounds caused by severe burns, deep tissue animal bites, skin tears and wounds caused by cutting or piercing instruments $[8,9]$.

Numerous wound dressing biomaterials are available currently in the market. However, majority of them are still taking quite a significant amount of time to heal the wounds [2, 4]. With the discovery of using silk protein as a biomaterial, researchers are creating new avenues to deliver the therapies for wound management [8, 9]. In this article, an effort has been put forth to highlight the recent studies going on in the area of silk fibroin based wound management with a compelling discussions on its effectiveness. Kashi is one of the most famous religious destination of India with millions of peoples visiting this great place every year. Thus, attainment of such a helpful wound management process is extremely helpful for Kashi which is as per the UN SDG goal 3 along with in line of Smart Kashi initiative.

\section{Skin repair-A multi step process:-}

Skin repair is a complex multistep process having involvement of a series of biochemical process. To design an appropriate wound dressing, thorough knowledge of these steps are extremely crucial [1, 10, 3]. The healing process consists of the following, overlapping stages; homeostasis, inflammation, proliferation, and remodeling [1, 3, 11]. Homeostasis stage is actually the immediate response of the body to an injury where fibrin develops a temporary barrier to stop blood loss at the wound site [12]. Inflammation stage usually persist form 24 h to 4-6 days when neutrophils and macrophages that sweeps the foreign particles and tissue debris from the wound bed [13]. Fibroblasts and myofibroblasts stimulated by cytokines and enzymes lead the wound exudates to provide essential moisture for the recovery [11]. New extracellular matrix (ECM) is formed in the proliferation phase when epithelialization occurs and newly formed granulation tissue begins to fill the wound area. Lastly, during the remodeling phase, a tight 3D network formation takes place by collagen-based cross-linking, thus, increasing the tensile strength of the new tissue $[12,3]$.

\section{Silk fibroin at a glance:-}

Many arthropods, such as spiders and silkworms produce silks in the epithelial cells of their specialized glands which are basically proteins having highly repetitive sequence, consisting mainly of glycine (43\%), alanine (30\%), and serine (12\%) - [GAGSGA]n motifs, arranged in -sheets regions embedded in an amorphous matrix [14, 3] and confer high toughness and elasticity [2, 4]. Silk of Bombyx mori silkworms contain two major protein components; fibroin and sericin. The fibroins are composed of mainly three types of protein fibers, a heavy chain of $350 \mathrm{kDa}$, a $30 \mathrm{kDa}$-glycoprotein, and a light chain of $25 \mathrm{kDa}$, the latter conferring hydrophilicity, water uptake ability and cell adhesion properties. Among the three protein fibres, light and heavy chains are connected by disulfide bonds, while the glue protein sericin coats the silk fibers. Silk fibroins have been successfully utilized for skin tissue engineering and wound healing applications for their high mechanical resistance, enzymatic-driven biodegradability and favorable cell attachment. Bombyx mori silkworm-derived fibroins are acquired from cocoon and separated from sericin by degumming in alkaline boiling water and following solubilization in hot LiBr solution [14, 3]. Upon modification of the helical chain arrangements into -sheets via alcohol treatment or water vapor annealing, the regenerated silk water soluble form, silk I, can be converted into insoluble silk II [15]. Moreover, by controlling the protein secondary structure, fibroin scaffolds' biodegradation can be properly modified in order to modulate the release of bioactive molecules [16], such as antibiotics, growth factors and antioxidants [17, 18]. Pignatelli and 
coworkers [19] encapsulated human platelet lysate into electrospun silk-PEO patches to prolong the growth factor shelf life and ease its handling during wound management. Versatile nature of proteins allow diverse processing techniques with consequent fabrication of multiple scaffold morphologies such as films, foams and sponges, gels, and fibrous matrices [20,21]. In recent time, electrospun fibroin has been extensively investigated for the design of anti-bacterial, anti-inflammatory and anti-oxidant patches [18,22]. To relieve a water-based electrospinning process, silk has been processed either in combination with natural polymers, such as cellulose, sericin [23], gelatin [24], chitosan [25], or mixed with synthetic materials, such as polyethylene oxide [26], polyvinyl alcohol etc. [17] which yielded great results in developing effective wound healing management $[2,4]$.

\section{Efficacy of silk fibroin in wound management:-}

In recent era, multiple studies proved the efficacy of silk fibroin in wound management $[2,4]$. It has been reported that a novel TEMPO-oxidized cellulose nanofiber (TOCN)-silk fibroin scaffold is significantly improved wound healing in an in vitro study model using primary fibroblast cells [27]. It has also been reported that the silk fibroin isolated from non-mulberry silkworm variety possesses cell binding motifs in the protein sequence which aid in better attachment of cells to the dressings, thereby recruiting cells for faster healing. In the initial trial in rabbit model it has been observed that the wounds are getting healed within 10-14 days compared to common surgical dressing (20 days) [17]. It has also been found that the silk dressing promoted more cellular proliferation and cellular recruitment towards the wound bed and accelerated the healing process. The wound dressings interacted with fibroblast cells of the wound thus led to the secretion of extracellular matrix (ECM) components like collagen and elastin. Moreover, the regular deposition of ECM fibers further helped in preventing the scarring of wounded tissue. This shows that the wound dressings can acte as an instructive platform for ideal skin regeneration [17]. Several researches are going on throughout the world for wound management involving natural products, syntheticnatural agents' combination etc. To that end, it has been found that blending silk fibroin with other components improved the properties of the resulting material to a great extent which also allows the modulation of biodegradation and release rates. In one such study, researchers developed silk fibroin/keratin films incorporating a synthetic inhibitor of elastase to control the high levels of this enzyme produced in a chronic wound environment $[21,28,29]$. Moreover, silk fibroin/alginate sponges demonstrate a higher healing effect than both components acting individually [21, 28, 29]. Silk/PVA mats loaded with Ciprofloxacin and epidermal growth factors [17] can enhance human dermal fibroblasts and keratinocytes proliferation in vitro, and favor re-epithelization, mature collagen deposition and complete wound closure at 14 days as found in an in vivo wound healing rabbit model. In a different work, Ju and coworkers [30] investigated the intrinsic anti-inflammatory effects of a porous fibroin/PEO electrospun nanomatrix in a mice burn-model, where down-regulation of pro-inflammatory cytokines IL-1a and IL-6 have been observed. Strontium loaded silk fibroin/sodium alginate (SF/SA) blend films can also be used as a promising biomaterial for wound dressing applications [31]. Moreover, people have also used fenugreek incorporated silk fibroin nanofiber mats which acted as antioxidant scaffolds in wound healing applications [32]. To accelerate the wound healing process, liposomes can be a very useful tool. It has been found that the liposome with SF hydrogel core can act as a potential carrier as growth factors for wound healing [33].

\section{Conclusion:-}

The wounds are of diverse types and the mechanism of wound healing is a complex process. Thus, selection of suitable dressing materials is of utmost importance. For biodegradable natural materials, their degradation also needs to follow the dynamics of the wound repair, guaranteeing the physiological healing evolution, and releasing active principles whenever required. In that line, proper consideration should be put onto the environmental sustainability of these biomaterials in terms of green chemistry fabrication approaches, and complete biodegradation without harmful by-products. Though, several reviews on traditional wound dressing biomaterials have been extensively studied and published worldwide, in this mini-review an effort has been taken to highlight the effectiveness of silk fibroin in wound management because of its multifarious potentials which will help further researches in this field to come up with a more high cutting edge product with suitable validation and enrich the medical science to serve people better.

\section{Conflict of interest:-}

There is no conflict of interest among authors.

\section{Acknowledgement:-}

This work is supported by HP CSR Initiative, catalyzed by Drstikona and implemented by Nalanda Foundation. 


\section{References:-}

1. Gurtner GC, Werner S, Barrandon Y, Longaker M. Wound repair and regeneration. Nature. 2008; 453:314-321.

2. Erfurt-Berge C, Renner R. Recent developments in topical wound therapy: impact of antimicrobiological changes and rebalancing the wound milieu. Biomed Res Int. 2014; 2014:819525.

3. Suarato G, Bertorelli R, Athanassiou A. Borrowing From Nature: Biopolymers and Biocomposites as Smart Wound Care Materials. Front Bioeng Biotechnol. 2018; 6:137.

4. Farokhi M, Mottaghitalab F, Fatahi Y, Khademhosseini A, Kaplan DL. Overview of Silk Fibroin Use in Wound Dressings. Trends Biotechnol. 2018; 36:907-922.

5. Sen CK, Gordillo GM, Roy S, Kirsner R, Lambert L, Hunt TK, et al. Human skin wounds: a major and snowballing threat to public health and the economy. Wound Repair Regen. 2009; 17:763-771.

6. Moura LI, Dias AM, Carvalho E, De Sousa HC. Recent advances on the development of wound dressings for diabetic foot ulcer treatment: a review. Acta Biomater. 2013; 9:7093-7114.

7. Guo S, Dipietro LA. Factors affecting wound healing. J. Dent. Res. 2010; 89:219-229.

8. Schneider A, Wang XY, Kaplan DL, Garlick JA, Egles C. Biofunctionalized electrospun silk mats as a topical bioactive dressing for accelerated wound healing. Acta Biomater. 2009; 5:2570-2578.

9. Khademhosseini A, Peppas NA. Micro- and nanoengineering of biomaterials for healthcare applications. Adv Healthc Mater. 2013; 2:10-12.

10. Pereira RF, Barrias CC, Granja PL, Bartolo PJ. Advanced biofabrication strategies for skin regeneration and repair. Nanomedicine. 2013; 8:603-621.

11. Das S, Baker AB. Biomaterials and nanotherapeutics for enhancing skin wound healing. Front Bioeng Biotechnol. 2016; 4:82.

12. Sinno H, Prakash S. Complements and the wound healing cascade: an updated review. Plastic Surg Int. 2013; 2013:146764.

13. Broughton G, Janis JE, Attinger CE. Wound healing: an overview. Plast Reconstr Surg. 2006; 117(Suppl. 7):1eS-32e-S.

14. Reimers K, Liebsch C, Radtke C, Kuhbier JW, Vogt PM. Silks as scaffolds for skin reconstruction. Biotechnol Bioeng. 2015; 112:2201-2205.

15. Hu X, Shmelev K, Sun L, Gil ES, Park SH, Cebe P, et al. Regulation of silk material structure by temperaturecontrolled water vapor annealing. Biomacromolecules. 2011; 12:1686-1696.

16. Hofmann S, Foo CT, Rossetti F, Textor M, Vunjak-Novakovic G, Kaplan DL, et al. Silk fibroin as an organic polymer for controlled drug delivery. J Control Release. 2006; 111:219-227.

17. Chouhan D, Chakraborty B, Nandi SK, Mandal BB. Role of non-mulberry silk fibroin in deposition and regulation of extracellular matrix towards accelerated wound healing. Acta Biomater. 2017; 48:157-174.

18. Lin S, Chen M, Jiang H, Fan L, Sun B, Yu F, et al. Colloids and surfaces B : biointerfaces green electrospun grape seed extractloaded silk fibroin nanofibrous mats with excellent cytocompatibility and antioxidant effect. Colloids Surf B Biointerf. 2016; 139:156-163.

19. Pignatelli C, Perotto G, Nardini M, Cancedda R, Mastrogiacomo M, Athanassiou A. Electrospun silk fibroin fibers for storage and controlled release of human platelet lysate. Acta Biomater. 2018; 73: 365-376.

20. Srivastava CM, Purwar R, Kannaujia R, Sharma D. Flexible silk fibroin films for wound dressing. Fibers Polym. 2015; 5:1020-1030.

21. Roh D-H, Kang S-Y, Kim J-Y, Kwon Y-B, Young Kweon H, Lee K-G, et al. Wound healing effect of silk fibroin/alginate-blended sponge in full thickness skin defect of rat. J Mater Sci Mater Med 2006;17:547-52.

22. Yang X, Fan L, Ma L, Wang Y, Lin S, Yu F, et al. (2017). Green electrospun Manuka honey/silk fibroin fibrous matrices as potential wound dressing. Mater Design. 2017; 119:76-84.

23. Guzman-Puyol S, Heredia-Guerrero JA, Ceseracciu L, Hajiali H, Canale C, Scarpellini A, et al. Low-cost and effective fabrication of biocompatible nanofibers from silk and cellulose-rich materials. ACS Biomater Sci. 2016; Eng. 2:526-534.

24. Shan YH, Peng LH, Liu X, Chen X, Xiong J, Gao JQ. Silk fibroin / gelatin electrospun nanofibrous dressing functionalized with astragaloside IV induces healing and anti-scar effects on burn wound. Int J Pharm. 2015; 479:291-301.

25. Cai ZX, Mo XM, Zhang KH, Fan LP, Yin AL, He CL, et al. Fabrication of chitosan/silk fibroin composite nanofibers for Wound-dressing applications. Int J Mol Sci. 2010; 11:3529-3539.

26. Chutipakdeevong J, Ruktanonchai UR, Supaphol P. Process optimization of electrospun silk fibroin fiber mat for accelerated wound healing. J Appl Polym Sci. 2013; 130:3634-3644. 
27. Shefa AA, Amirian J, Kang HJ, Bae SH, Jung HI, Choi HJ, Lee SY, Lee BT. In vitro and in vivo evaluation of effectiveness of a novel TEMPO-oxidized cellulose nanofiber-silk fibroin scaffold in wound healing. Carbohydr Polym. 2017; 177:284-296.

28. Vasconcelos A, Pêgo AP, Henriques L, Lamghari M, Cavaco-Paulo A. Protein matrices for improved wound healing: elastase inhibition by a synthetic peptide model. Biomacromolecules 2010;11:2213-20.

29. Vasconcelos A, Gomes AC, Cavaco-Paulo A. Novel silk fibroin/elastin wound dressings. Acta Biomater. 2012; 8:3049-3060.

30. Ju HW, Lee OJ, Lee JM, Moon BM, Park HJ, Park YR, et al. Wound healing effect of electrospun silk fibroin nanomatrix in burn-model. Int J Biol Macromol. 2016; 85:29-39.

31. Li S, Li L, Guo C, Qin H, Yu X. A promising wound dressing material with excellent cytocompatibility and proangiogenesis action for wound healing: Strontium loaded Silk fibroin/Sodium alginate (SF/SA) blend films. Int J Biol Macromol. 2017; 104(Pt A):969-978.

32. Selvaraj S, Fathima NN. Fenugreek Incorporated Silk Fibroin Nanofibers-A Potential Antioxidant Scaffold for Enhanced Wound Healing. ACS Appl Mater Interfaces. 2017; 9:5916-5926.

33. Xu HL, Chen PP, ZhuGe DL, Zhu QY, Jin BH, Shen BX, Xiao J, Zhao YZ. Liposomes with Silk Fibroin Hydrogel Core to Stabilize bFGF and Promote the Wound Healing of Mice with Deep Second-Degree Scald. Adv Healthc Mater. 2017; 6(19). 